University of Nebraska - Lincoln

DigitalCommons@University of Nebraska - Lincoln

\title{
8-15-1995
}

\section{Mercury surface-plasmon dispersion: Experiment and theory}

\author{
Bong-Ok Kim \\ University of Pennsylvania \\ Geunseop Lee \\ University of Pennsylvania- Philadelphia \\ E. Ward Plummer \\ University of Tennessee, Knoxville, wplummer@phys.Isu.edu
}

Peter A. Dowben

University of Nebraska-Lincoln, pdowben@unl.edu

A. Liebsch

Institut fur Festkorperforschung, Forschungszentrum Julich, Julich, Germany

Follow this and additional works at: https://digitalcommons.unl.edu/physicsdowben

Part of the Physics Commons

Kim, Bong-Ok; Lee, Geunseop; Plummer, E. Ward; Dowben, Peter A.; and Liebsch, A., "Mercury surfaceplasmon dispersion: Experiment and theory" (1995). Peter Dowben Publications. 51.

https://digitalcommons.unl.edu/physicsdowben/51

This Article is brought to you for free and open access by the Research Papers in Physics and Astronomy at DigitalCommons@University of Nebraska - Lincoln. It has been accepted for inclusion in Peter Dowben Publications by an authorized administrator of DigitalCommons@University of Nebraska - Lincoln. 


\title{
Mercury surface-plasmon dispersion: Experiment and theory
}

\author{
Bong-Ok Kim* and Geunseop Lee* \\ Department of Physics, University of Pennsylvania, Philadelphia, Pennsylvania 19104 \\ E. W. Plummer \\ Department of Physics and Astronomy, University of Tennessee, Knoxville, Tennessee 37996 \\ and Solid State Division, Oak Ridge National Laboratory, P.O. Box 2008, Oak Ridge, Tennessee 37831-6057 \\ P. A. Dowben \\ Department of Physics, University of Nebraska-Lincoln, Lincoln, Nebraska 68588-0111 \\ A. Liebsch \\ Institut für Festkörperforschung, Forschungszentrum Jülich, 24825 Jülich, Germany \\ (Received 7 December 1994; revised manuscript received 17 March 1995)
}

\begin{abstract}
Momentum-resolved inelastic electron spectroscopy has been utilized to measure the surfaceplasmon dispersion and damping for thick $\mathrm{Hg}$ films grown onto a $\mathrm{Cu}(100)$ substrate. $\mathrm{Hg}$ has been investigated since it offers an opportunity to examine the effects of the crystal potential on the surface-plasmon dispersion, and to correlate the measurements on a surface with those for collective excitations in clusters. The effects of the electronic band structure of $\mathrm{Hg}$ can be seen in the energy and width of the surface plasmon as a function of momentum transfer $q_{\|}$. Dynamical response calculations were performed for a semi-infinite electron gas where several important aspects of the crystal potential are taken into account in an average manner. These effects are shown to lead to an overall lowering of the plasma frequency, to a flattening of the momentum dispersion, and to a sizable broadening of the plasmon linewidth relative to the behavior obtained for the simple metals. Even though the band structure effects in $\mathrm{Hg}$ are significant, there is a quantitative correlation between the normalized energy and width of the surface plasmon as a function of $q_{\|}$and the Mie resonance seen in $\mathrm{Hg}$ clusters as a function the inverse of the radius $R$.
\end{abstract}

\section{INTRODUCTION}

Significant progress has been made in understanding the physical nature of collective electronic excitations at metal surfaces, since the concept of a surface plasmon was introduced nearly forty years ago by Ritchie. ${ }^{1}$ Major advances occurred in the 1970's when Harris and Griffin, ${ }^{2}$ Flores and Garcia-Moliner, ${ }^{3}$ and Feibelman ${ }^{4}$ showed that the initial slope of the surface-plasmon dispersion with parallel wave vector has a very simple geometrical meaning: it is given by the negative of the centroid position of the fluctuating charge density associated with the surface plasmon. Feibelman also calculated this centroid position within the random phase approximation (RPA), utilizing accurate ground-state electronic properties based on the Lang-Kohn jellium model. ${ }^{5}$ These calculations demonstrated that, for all jellium surfaces at typical densities, the charge centroid of the surface-plasmon is located outside the metal surface, giving a negative linear dispersion at small wave vectors. Experimental confirmation of this prediction came in 1989 when Tsuei measured the surface-plasmon dispersion for $\mathrm{Na}$ and $\mathrm{K} .{ }^{6}$ At about the same time, the complete spectrum of electronic excitations at jellium surfaces was calculated ${ }^{7}$ for arbitrary parallel momenta within the time-dependent local-density approximation (TDLDA). ${ }^{8}$ These results were in good overall agreement not only with the full dispersion of the surface plasmon, but also with that of the so-called multipole surface-plasmon mode, which occurs at slightly higher frequencies.

A more detailed comparison of the surface-plasmon data of the simple metals with the dynamical response calculations based on the jellium model reveals three slight, but nevertheless persistent, systematic discrepancies: First, there is, of course, a small overestimate of all frequencies, because of the neglect of core polarization. Second, the width of the measured plasmon lines tends to be considerably larger than the calculated ones, in particular, at small momenta, where the jellium model predicts that the width should decrease linearly to zero. Third, at small momenta, the TDLDA tends to overestimate the magnitude of the linear slope of the monopole plasmon, so that the data appear to agree better with the RPA predictions. On the other hand, the surface plasmon at larger momenta is better described within the TDLDA.

There are several possible reasons for these systematic differences between theory and experiment. (1) The jellium model misses any effect due to the crystal potential of the solid. (2) The use of the LDA in the groundstate calculation does not reproduce the image potential. (3) The theoretical schemes used so far to calculate the dynamical response are approximate: the TDLDA includes a local adiabatic exchange-correlation correction (in a region where the density is changing rapidly), while 
the RPA ignores exchange-correlation effects in the response entirely.

The focus of this study of the surface-plasmon dispersion of solid $\mathrm{Hg}$ is to investigate various effects arising from the crystal potential. We have chosen $\mathrm{Hg}$, because the optical data show that band structure effects will be important, yet the effects should not be as dominant as they are in Ag. In addition, there are published data for the collective excitation of $\mathrm{Hg}$ clusters as a function of cluster radius ${ }^{9}$ that can be compared to the measurements of the surface-plasmon dispersion.

The theory used in the present work introduces three important improvements related to the influence of the crystal potential that go beyond the scheme employed previously for the simple metals: (1) Instead of the standard Lang-Kohn jellium model, we use the so-called stabilized jellium model, ${ }^{10}$ which yields more realistic surface density profiles, work functions, and surface energies. This improvement is achieved by taking the ionic pseudopotentials into account in an average manner. (2) The effect of bulk optical interband transitions involving the conduction band is included by evaluating the dynamical surface response at complex frequencies whose imaginary component is related to the imaginary part of the bulk dielectric constant. (3) The core polarization due to the $\mathrm{Hg} 5 d$-core levels is described in terms of a polarizable medium, which partially screens the self-consistent dynamical Coulomb interaction between the conduction electrons. The mutual $s$ - $d$ polarization had recently been shown by Liebsch ${ }^{11,12}$ to lead to the positive dispersion of the Ag surface plasmons.

The attractive feature of these theoretical improvements beyond the standard jellium model is that they retain the computational simplicity of one-dimensional models. Yet, as the results of our self-consistent TDLDA response calculations demonstrate, the above average treatment of lattice effects captures the essential physics and leads to remarkable agreement with the experimental data. The main results of our work are as follows. (1) The shallow $\mathrm{Hg} 5 d$-core levels cause an overall lowering of the surface-plasmon frequencies by about $12 \%$ relative to the free-electron values. (2) The core polarization and interband transitions lead to a significant flattening of the momentum dispersion compared to the dispersion obtained for the simple metals. The linear coefficient of the dispersion is still negative, but much smaller than in the absence of the lattice effects. Accordingly, the minimum of the dispersion curve is shifted to smaller momenta. (3) The multipole surface-plasmon mode is suppressed. (4) The width of the $\mathrm{Hg}$ surface plasmon at small parallel wave vectors is well reproduced by the damping based on the imaginary part of the bulk dielectric function. At larger $q_{\|}$, the damping due to bulk absorption processes must be reduced in order to account for the smaller penetration depth of the induced electric field.

The main conclusion from these results is that lattice effects must be taken into account, at least in the average sense as proposed above, in order to achieve an adequate understanding of the properties of collective modes at realistic metal surfaces. Additional effects might arise from nonlocal corrections to the ground-state potential and from nonadiabatic as well as nonlocal corrections to the induced potential. These corrections should not, however, be considered independently of the modifications associated with the crystal potential.

A surface-plasmon mode, the best known normal mode at the surface of a metal, is a consequence of the requirement that the normal component of the displacement vector be continuous in passing across the interface between the vacuum and the metal. ${ }^{1}$ Consequently, the energy of this mode is determined by the properties of the bulk, i.e., when $\varepsilon=-1$. If $\varepsilon$ can be represented by a Drude equation $\varepsilon_{d}(\omega)=1-\omega_{p}^{2} / \omega^{2},\left(\omega_{p}^{2}=4 \pi n e^{2} / m\right)$, then the energy of the surface plasmon is $\omega_{\text {sp }}=\omega_{p} / \sqrt{2}$. The dynamical response of the electrons at the surface affects the properties of the surface plasmon for $q_{\|} \neq 0$, such as the dispersion and line shape. In general, the properties of the normal modes at a surface are defined by the poles of the surface response function $g\left(q_{\|}, \omega\right)$, which according to Persson and Zaremba ${ }^{13}$ can be written as

$$
g\left(q_{\|}, \omega\right)=\int d z e^{q z} \delta n\left(q_{\|}, z, \omega\right),
$$

where $\delta n$ is the charge induced by the external field. The response function can be expanded in the small $q_{||}$limit $^{14}$ giving

$$
g\left(q_{\|}, \omega\right)=\frac{[\varepsilon(\omega)-1]\left[1+q_{\|} d(\omega)\right]}{\varepsilon(\omega)+1-[\varepsilon(\omega)-1] q_{\|} d(\omega)},
$$

where the function $d(\omega)$ characterizes the surface response to the external field and is given by ${ }^{14}$

$$
d(\omega)=\frac{\int d z z \delta n(z, \omega)}{\int d z \delta n(z, \omega)}
$$

Thus, $d(\omega)$ is the first moment of the induced charge and is a complex function of the frequency. If the dielectric response is given by the Drude formula, then Eq. (2) reduces to $^{2-4}$

$$
\omega_{\mathrm{sp}}=\omega_{\mathrm{sp}}\left(q_{\|}=0\right)\left[1-\frac{q_{||} \operatorname{Re}\left[d\left(\omega_{\mathrm{sp}}\right)\right]}{2}+\cdots\right] .
$$

For all simple metals, the first moment of the induced charge is outside of the surface so that $\operatorname{Re}\left[d\left(\omega_{\mathrm{sp}}\right)\right]$ is positive and the dispersion of the surface plasmon for small $q_{\|}$is negative. ${ }^{6,7,15}$

As stated above, the main issue addressed in this paper is an assessment of the effect of the crystal potential on the properties of the surface plasmon. Let us focus now on two manifestations of the lattice potential: interband transitions involving the conduction band, and the polarization of shallow bound states. In the bulk, the deviation from free-electron behavior caused by these effects can be qualitatively described by expressing the macroscopic dielectric function as $\varepsilon(\omega)=\varepsilon_{1}(\omega)+i \varepsilon_{2}(\omega)$ with $\varepsilon_{1}(\omega)=\varepsilon_{d}(\omega)+\varepsilon_{b}(\omega) . \varepsilon_{d}$ represents the real part of the Drude response of the conduction electrons, $\varepsilon_{2}$ describes the interband contribution of the conduction band and the imaginary part of the Drude term, and $\varepsilon_{b}$ is the real part of the band structure contribution, due to interband 

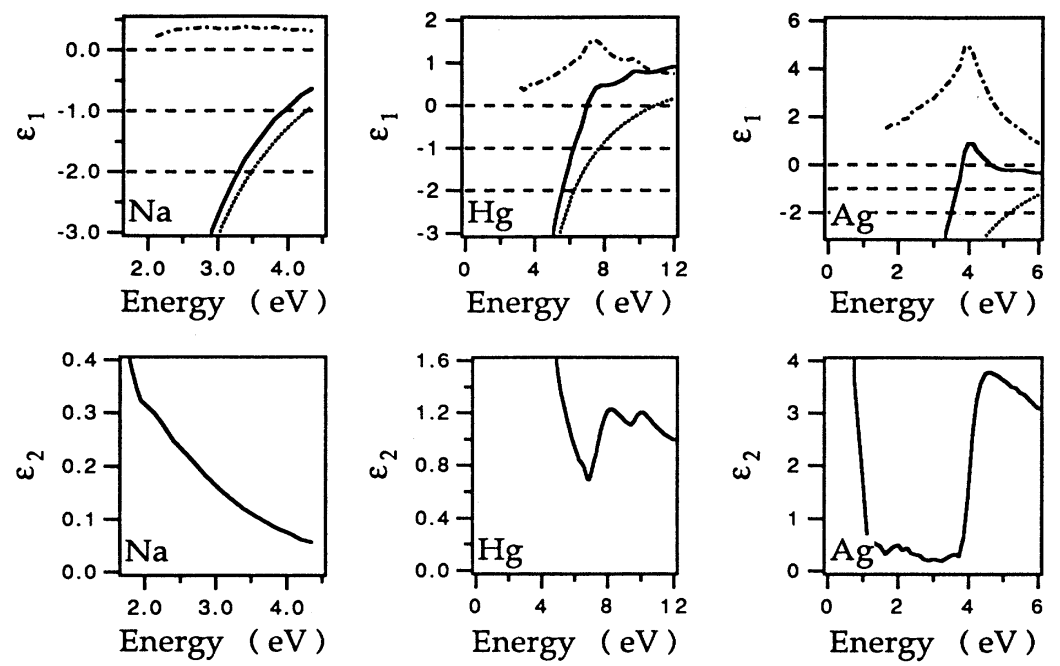

FIG. 1. A plot of the $\varepsilon_{1}$ (solid line), the Drude formula (dotted line), and the calculated $\varepsilon_{b}$ (dot-dashed line) for $\mathrm{Na}$ (Ref. 17), $\mathrm{Hg}$ (Ref. 18), and Ag (Ref. 19). The bottom panel for each material is the frequency dependence of $\varepsilon_{2}$. transitions and occupied core states. ${ }^{16}$ The importance of the band structure in different materials is illustrated in Fig. 1, where at the top the measured frequency dependence of $\varepsilon_{1}$ is compared to the Drude formula (dotted line), and the calculated $\varepsilon_{b}$ is plotted (dot-dashed) for three different materials, $\mathrm{Na}$, liquid $\mathrm{Hg}$, and $\mathrm{Ag}$. The peak in $\varepsilon_{b}$ for $\mathrm{Hg}$ at $\sim 7.5 \mathrm{eV}$ and for $\mathrm{Ag}$ at $\sim 4 \mathrm{eV}$ is a consequence of the presence of $d$-band $(\mathrm{Ag})$ or $d$-core levels (Hg) below the Fermi energy. In the region of the surface plasmon $\varepsilon_{b}$ is $\sim 0.3$ for $\mathrm{Na}, \sim 1$ for liquid $\mathrm{Hg}$, and $\sim 4$ for Ag.

In Sec. III, we will describe the results of nonlocal surface response calculations that include the band structure contributions $\varepsilon_{2}(\omega)$ and $\varepsilon_{b}(\omega)$ in a self-consistent manner. In the long-wavelength limit, these effects are given by Eq. (2), provided the function $d(\omega)$ is evaluated in the presence of the same band structure effects as the surface response function $g(q, \omega)$. If $\varepsilon_{2}, \varepsilon_{b}$, and $d(\omega)$ are slowly varying functions of $\omega$ near $\omega_{\mathrm{sp}}$, an expansion for the energy and width of the surface plasmon as a function of $q_{\|}$can be derived

$$
\begin{aligned}
& \frac{\omega}{\omega_{\mathrm{sp}}}=1-\frac{q_{\|}}{2}\left[d_{1}+\frac{\varepsilon_{2} d_{2}-\varepsilon_{b} d_{1}}{2+\varepsilon_{b}}\right], \\
& \frac{\Delta \omega}{\omega_{\mathrm{sp}}}=\frac{\varepsilon_{2}}{2+\varepsilon_{b}}+q_{\|}\left[d_{2}-\frac{\varepsilon_{b} d_{2}+\varepsilon_{2} d_{1}}{2+\varepsilon_{b}}\right],
\end{aligned}
$$

where $d(\omega)=d_{1}+i d_{2}, \omega_{\mathrm{sp}}^{2}=\omega_{p}^{2} /\left(2+\varepsilon_{b}\right)$, and $\omega_{p}$ is the free-electron energy of the bulk plasmon.

In the case of $\mathrm{Na}$, the constant value of $\varepsilon_{b}$ due to core polarization is quite weak and has little effect on the dispersion of the surface plasmon. On the other hand, the influence of the $d$ bands is so severe for Ag (Refs. 20-22) that the dispersion differs from that of the simple metals in three fundamental aspects: $(1)$ the frequency $(\sim 3.7$ $\mathrm{eV})$ is much lower than expected for the $s$ electrons alone $(\sim 6.5 \mathrm{eV}),(2)$ the dispersion is positive rather than negative even at small $q_{\|}$, and (3) the dispersion differs for the three low-index faces and is anisotropic on $\mathrm{Ag}(110)$. It would therefore be completely inappropriate, to use the $d(\omega)$ functions derived for standard jellium surfaces to es- timate the small- $q$ behavior of the Ag surface plasmon. (In fact, this inconsistent procedure predicts a negative dispersion for $\mathrm{Ag}$ in contrast to the data.) Instead, $d(\omega)$ must be reevaluated consistently in the presence of the band structure contributions. ${ }^{23}$

Although $\mathrm{Hg}$ is much closer to a free-electron metal than Ag, we will show below that it is nevertheless important to take into account the lattice effects on the function $d(\omega)$. Thus, the small- $q$ behavior of the surface plasmon and its width can be obtained from Eqs. (5) and (6), but the $d(\omega)$ function should not be approximated by that of a free-electron gas.

The second objective of this study is to explore the relationship between the dispersion of the surface plasmon and the size dependence of the Mie resonance in clusters. ${ }^{12,24,25}$ For spherical geometry, the classical solution to Maxwell's equation gives a normal mode (Mie resonance) at the frequency, where $\varepsilon=-2$, a result derived by Mie in $1908 .^{26}$ For finite particle radii, deviations from this limit occur due to the spill out of the charge into the vacuum. Apell and Ljungbert ${ }^{24,25}$ were the first to show that this frequency shift is closely related to the linear dispersion of the surface plasmon at small $q_{\|}$. While the simple metals show a redshift of the Mie resonance with decreasing radius ${ }^{27}$ (just like the surface plasmon shifts initially downwards with increasing $\left.q_{\|}\right)$, the size dependence for Ag particles exhibits a blueshift ${ }^{28}$ (analogous to the positive dispersion of the Ag surface plasmon).

In the present work, we demonstrate that also in the case of $\mathrm{Hg}$ there is a remarkably close correspondence between the momentum dispersion of the surface plasmon and the cluster size dependence of the Mie resonance. ${ }^{9}$ This correspondence applies to both the normalized energies of these modes and their width. The $\mathrm{Hg}$ data will compliment equivalent comparisons for $\mathrm{K},{ }^{29} \mathrm{Na},{ }^{30}$ and Ag. ${ }^{12,28}$

\section{EXPERIMENT}

The electron-energy-loss spectra (ELS) were measured using a LK-2000 angle-resolved electron-energy-loss spec- 
trometer mounted in a standard UHV chamber operated at a pressure of $\sim 1 \times 10^{-10}$ torr. $\mathrm{Hg}$ was admitted to the chamber with a doser using the vapor of triply distilled $\mathrm{Hg}$. Films were grown on a clean and annealed $\mathrm{Cu}(100)$ sample held at $\sim 100 \mathrm{~K}$, with the thickness being determined by the time required to complete monolayer. The films used in this experiment were approximately 60 layers thick. The smoothness of the $\mathrm{Hg}$ films was monitored by the intensity and angular width of the specularly reflected electron beam. Condensation of a 60 layer $\mathrm{Hg}$ film at $100 \mathrm{~K}$ onto $\mathrm{Cu}(100)$ caused a fivefold reduction in the intensity of the elastically scattered beam, but annealing to $200 \mathrm{~K}$ restored the elastic intensity to approximately $1 / 2$ of clean $\mathrm{Cu}$. After annealing the $\mathrm{Hg}$ films, the angular profile of the elastic beam was $2.5^{\circ}$, similar to the angular width from clean $\mathrm{Cu}$.

The incident energy $E_{0}$ in these experiments was 45 $\mathrm{eV}$, and the incident angle, $\theta_{i}, 60^{\circ}$ with respect to the surface normal was used. The momentum transfer parallel to the surface is given by

$$
q_{\|}=\left(4 \pi m / \hbar^{2}\right)^{1 / 2}\left(\sqrt{E_{0}} \sin \theta_{i}-\sqrt{E_{s}} \sin \theta_{s}\right)
$$

where $\theta_{s}$ is the angle of the scattered electrons and $E_{s}$ is the energy of the inelastically scattered electrons, $E_{s}=$ $E_{0}-\Delta E . \Delta E$ is the energy loss. $q_{\|}$is varied by changing $\theta_{s}$ for a fixed value of $\theta_{i}$ and $E_{0}$. The energy resolution in these experiments was $\sim 30 \mathrm{meV}$ and $q_{||}$resolution was $0.1 \AA^{-1}$ corresponding to the $2.5^{\circ}$ angular resolution.

Figure 2 shows loss spectra taken on a $\mathrm{Hg}$ film grown on $\mathrm{Cu}(100)$ at $90 \mathrm{~K}$, annealed to $200 \mathrm{~K}$ for $10 \mathrm{~min}$ and then cooled to $90 \mathrm{~K}$, where the data was recorded. The peak in the loss spectra near $7 \mathrm{eV}$ is the surface plasmon. For comparison, we have calculated the surface loss function $\operatorname{Im}(\varepsilon+1)^{-1}$ for $q_{\|}=0$, using the measured liquid $\mathrm{Hg}$ dielectric constants. ${ }^{18}$ The calculated loss function for liquid $\mathrm{Hg}$ has a peak at $6.3 \mathrm{eV}$ and a full width at half maximum (FWHM) of $\sim 1.0 \mathrm{eV}$, while our data which is for solid $\mathrm{Hg}$ shows a surface plasmon at an energy of $6.83 \mathrm{eV}$ for $q_{\|}=0$, with a width of $\sim 1.1 \mathrm{eV}$. It is well known that there is an appreciable difference in the dielectric constants for liquid and solid $\mathrm{Hg}$, especially in the energy range appropriate for band transition, $<3$ eV. ${ }^{18,31,32}$ The problem is that the published data for solid $\mathrm{Hg}$ are inconsistent ${ }^{31,32}$ in the lower energy range of the bulk transitions. The optical data of Choyke et $a l .{ }^{31}$ gives a value for the surface plasmon $\left(\varepsilon_{1}=-1\right)$ of $5.8 \mathrm{eV}$, far too low compared to our data or that of Krauss and Gomer. ${ }^{33}$ We have used our data to obtain, $\varepsilon_{2}$ and $\varepsilon_{b}$ at the frequency of the surface plasmon $(6.83 \mathrm{eV})$ for solid $\mathrm{Hg}$, which are $\varepsilon=-1+i 0.41$ and $\varepsilon_{b}=0.56$. This can be compared to the measured values for liquid $\mathrm{Hg}^{18}$ at the same energy $(6.83 \mathrm{eV})$, which are $\varepsilon=-0.3+i 0.69$ and $\varepsilon_{b}=1.25$.

The energy and width of the surface-plasmon mode was extracted from the raw data by fitting to a single Lorentzian peak. As $q_{\|} \rightarrow 0$, the line shape is very asymmetric, presumably due to the excitation of the bulk plasmon and the multipole surface-plasmon mode. The analysis emphasized fitting the leading edge of the loss peak. Figure 3(a) displays the energy of the surfaceplasmon mode on $\mathrm{Hg}$ as a function of $q_{\|}$, where the solid line is the best fit quadratic function $\left(q_{\|}\right.$is in units of $1 / \AA)$,

$$
\hbar \omega_{\mathrm{sp}}\left(q_{\|}\right)=6.83\left(1-0.167 q_{||}+0.84 q_{\|}^{2}\right) \quad \mathrm{eV} .
$$

Since the $q_{\|}$resolution reduces the negative plasmon dispersion coefficient, our first-order coefficient of the dispersion is the lower bound of its intrinsic value.

It is useful to compare these results to what is expected from an electron gas with the density of $\mathrm{Hg}\left(r_{s}=2.65\right)$. The $q=0$ surface plasmon for this electron density should appear at an energy of $7.72 \mathrm{eV}$, and the dispersion $d\left(\omega_{\mathrm{sp}}\right) / 2$ [Eq. (4)] is $0.50 \AA$ in the TDLDA (Ref. 34) and $0.32 \AA$ using the RPA, ${ }^{14,34}$ where $\omega_{\text {sp }}$ is the free-electron value. It is conceivable that the experimental data for small $q$ could be explained by simple first-order perturbation. Adding the band structure through $\varepsilon_{b}$ and the damping through $\varepsilon_{2}$ to $d(\omega)$ of the free-electron gas model makes very little effect in the dispersion, because the last two terms in Eq. (5) almost cancel each other for $\mathrm{Hg}$. [The linear dispersion in Eq. (5) using $d(\omega)$ in TDLDA is $0.49 \AA$.] Therefore, again, we need to calculate $d(\omega)$

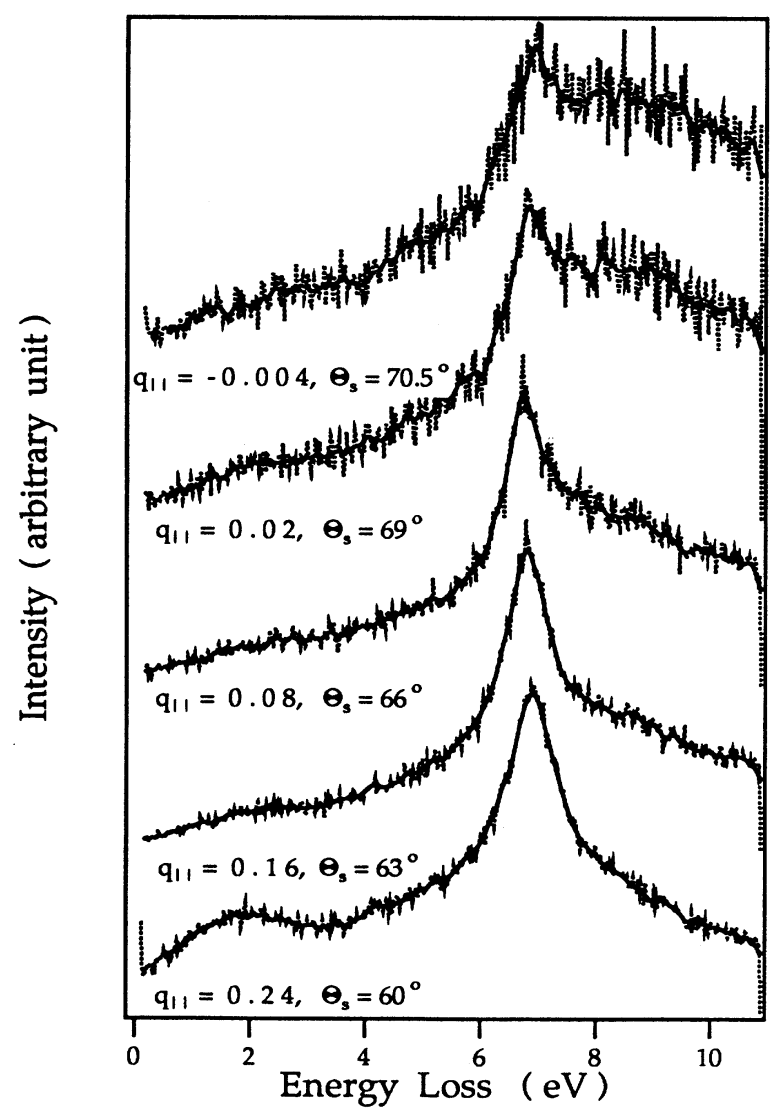

FIG. 2. Angular dependence of the electron-energy-loss spectra for thick $\mathrm{Hg}$ films. The incident energy is $45 \mathrm{eV}$ and the incident angle is $60^{\circ}$. The scattering angles and the corresponding $q_{\|}$values are presented. 

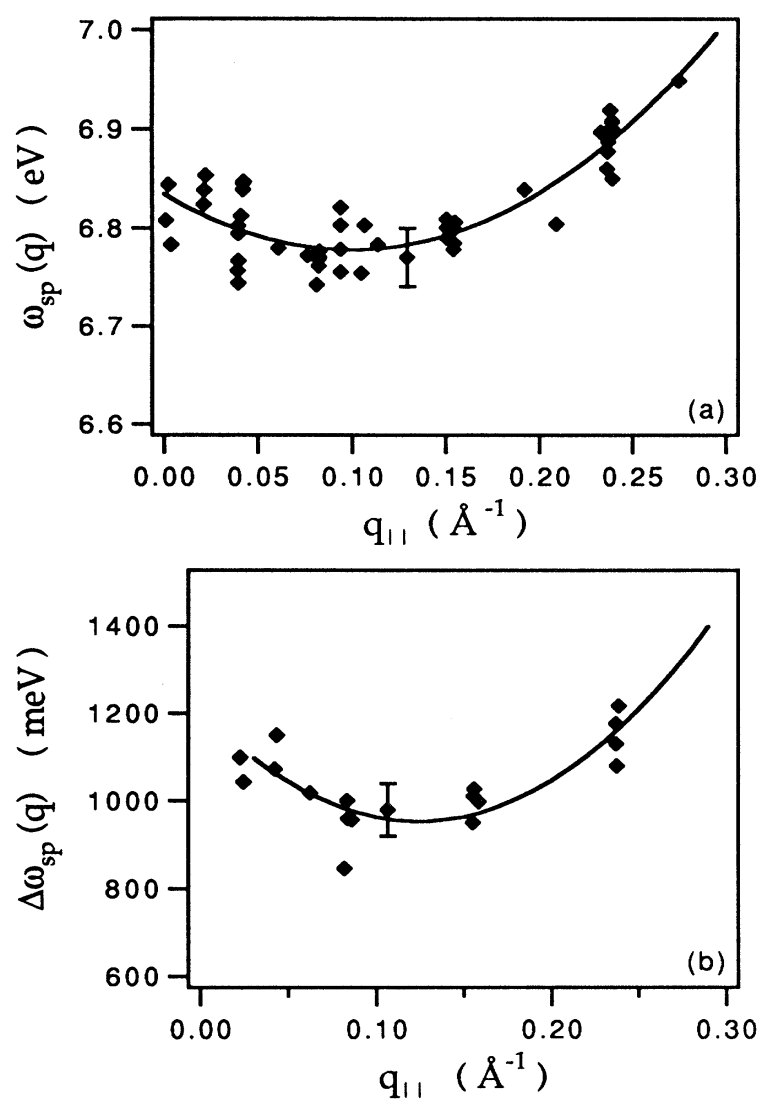

FIG. 3. The measured (a) dispersion and (b) linewidth for the surface plasmon of $\mathrm{Hg}$. The error bar of the plasmon energy is about $60 \mathrm{meV}$ and that of the width is about 100 meV, which come from Lorentzian fitting of the spectra in addition to the experimental error. The solid line is the best fit to the data.

in a self-consistent way including all lattice effects. In other words, the first-order perturbation approach with the standard jellium model does not work for $\mathrm{Hg}$, even though the band structure effects of $\mathrm{Hg}$ is significantly weaker than those of Ag.

The measured surface plasmon for $\mathrm{Hg}$ is $\sim 0.9 \mathrm{eV}$ lower ( $\sim 12 \%$ shifted $)$ in energy than expected from a freeelectron gas and the linear term of the dispersion is much smaller than the values calculated within the RPA or TDLDA for the jellium surface. Part of this difference is undoubtedly due to the presence of the occupied $5 d$-core levels approximately $8 \mathrm{eV}$ below the Fermi energy and due to the interband transitions involving the $6 s$ band of $\mathrm{Hg}$. Figure 1 showed the effect of this excitation on the dielectric constant. For comparison, the effects of the $d$ band on the collective excitations in Ag are much more dramatic. The surface plasmon of $\mathrm{Ag}$ is shifted from the free-electron value of $6.35 \mathrm{eV}$ down to $3.71 \mathrm{eV}$ $(42 \%$ shifted) and the dispersion is positive even at small $q_{\|}$. The reason is, of course, that the band structure contribution to the dielectric constant of $\mathrm{Ag}$ is much larger than for $\mathrm{Hg}$, with $\varepsilon_{b}\left(\omega_{s}\right) \simeq 4$.

The surface-plasmon broadening shown in Fig. 3(b) is about $1.1 \mathrm{eV}$, with a slight anomalous behavior at small $q_{\|}$, where the width first decreases. The finite momentum resolution makes a more negative width trends near $q_{||}$corresponding to the minimum plasmon energy. However, this effect on the linewidth is about $30 \mathrm{meV}$, which does not alter the overall trends. Similar behavior had also been observed for the surface plasmon on $\mathrm{Mg}(0001)$ (Ref. 15) and for $\operatorname{Ag}(111) .{ }^{35}$ If this phenomenon existed only on $\mathrm{Hg}$ and $\mathrm{Ag}$, it would be tempting to associate this narrowing with band structure effects. In the case of $\mathrm{Mg}$, however, these effects on the surface plasmon are negligible.

In the standard semi-infinite jellium system, the width of the surface plasmon at small $q_{\|}$increases linearly, with the coefficient determined by $\operatorname{Im} d\left(\omega_{s}\right) / 2$. For $r_{s}=2.65$, this coefficient is $0.82 \AA$ in the TDLDA, while the RPA yields $0.61 \AA .{ }^{34}$ At $q_{\|}=0.1 \AA^{-1}$, these values would amount to a width of $0.32 \mathrm{eV}$ (TDLDA) and $0.24 \mathrm{eV}$ (RPA), respectively. The large discrepancy to the data shown in Fig. 3(b) at small $q_{\|}$is clearly caused, at least in part, by transitions not accounted for in the standard jellium model, as is evident from the imaginary part of the bulk dielectric function.

We now turn to the comparison of these surfaceplasmon measurements with the $\mathrm{Hg}$ cluster data. ${ }^{9}$ According to Apell and Ljungbert, ${ }^{24,25}$ the size dependence of the Mie resonance frequency for spherical particles in the limit of large radii should be given by

$$
\frac{\omega_{M}(R)}{\omega_{M}(\infty)}=1-\frac{3}{3+\varepsilon_{b}} \frac{\operatorname{Re} d_{r}\left(\omega_{M}\right)}{R}
$$

where $\omega_{M}(\infty)$ is the Mie resonance for an infinitely large cluster defined by the spherical loss function $\operatorname{Im}(\varepsilon+2)^{-1}$, which gives $\omega_{M}^{2}(\infty)=\omega_{p}^{2} /\left(3+\varepsilon_{b}\right)$, with the free-electron energy of the bulk plasmon $\omega_{p}$. The above formula holds if the effect due to bound core levels described via $\varepsilon_{b}$ is taken into account. If both damping due to interband transitions and core polarization are included, Eq. (9) can be generalized, similar to Eq. (5),

$$
\frac{\omega_{M}(R)}{\omega_{M}(\infty)}=1-\frac{1}{R}\left[\operatorname{Re} d_{r}+\frac{\varepsilon_{2} \operatorname{Im} d_{r}-\varepsilon_{b} \operatorname{Re} d_{r}}{3+\varepsilon_{b}}\right] .
$$

We emphasize that these expressions are meaningful only if the function $d_{r}(\omega)$ is evaluated in the presence of the crystal effects described by $\varepsilon_{2}$ and $\varepsilon_{b}$. Thus, the same consistency argument applies here as for the surface plasmons. This is obvious, for example, in the case of $\mathrm{Ag}$ particles: if $\varepsilon_{b} \approx 4$ is included in Eq. (9), but $d_{r}(\omega)$ is taken from standard surface jellium calculations with $\operatorname{Red}\left(\omega_{M}\right)>0$, the dependence of the $\mathrm{Ag}$ Mie plasmon on $1 / R$ is predicted to be negative in conflict with $\mathrm{Ag}$ cluster data, which show a positive dependence. ${ }^{28}$

The second set of the terms multiplied by $1 / R$ almost cancels for $\mathrm{Hg}$ as they did in Eq. (5), therefore we have a simple form of Eq. (10) that can be compared to Eq. (4) directly:

$$
\frac{\omega_{M}(R)}{\omega_{M}(\infty)}=1-\frac{\operatorname{Re} d_{r}\left(\omega_{M}\right)}{R}
$$


$d_{r}(\omega)$ depends upon the charge spill out for the cluster and the response of that charge to the incident field at frequency $\omega$, just as in the case of a surface.

The correspondence between the surface-plasmon dispersion and the size dependence of the Mie resonance has been used to explain the redshift in the Mie resonance for $\mathrm{Na}^{30}$ and $\mathrm{K},{ }^{29}$ and the blueshift for $\mathrm{Ag}$ clusters. ${ }^{28}$ Figure 4 shows the normalized energy and width of the surface plasmon and of the Mie resonance. The agreement between the cluster data and the surface-plasmon dispersion is surprising, especially given the fact that existing theories are applicable for the comparison of surface plasmons at $q \rightarrow 0$ and clusters where $R \rightarrow \infty$. The similarities beyond this range need considerably more investigation, both experimentally and theoretically. The striking resemblance of these dispersions strongly suggests that the same physical processes govern the energy and width of the collective excitation in a spherical cluster and at a flat surface. This analogy seems to hold not only in the linear region (up to about $0.1 \AA$ ), but also at larger values of $q_{\|}$or $1 / R$.
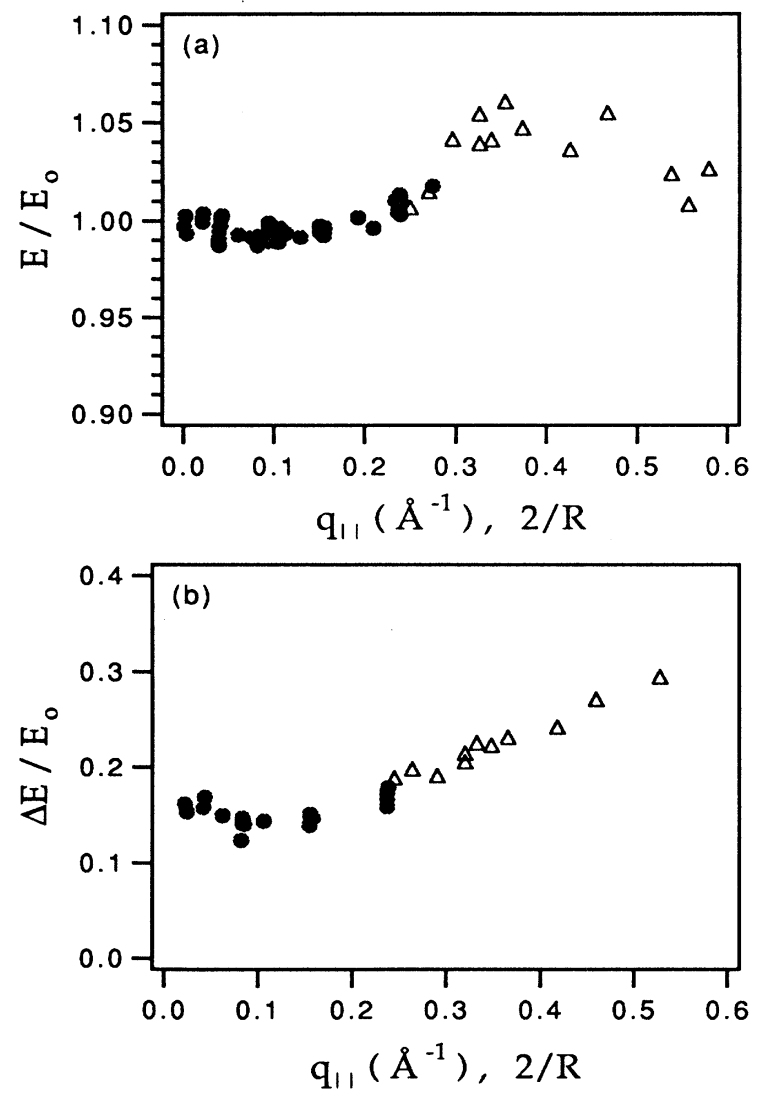

FIG. 4. A comparison of the normalized (a) energies and (b) linewidths for the surface plasmon (circle) as a function of $q_{\|}$and clusters (triangle) as a function of $2 / R$ (Ref. 9). Surface-plasmon energies are normalized by $6.83 \mathrm{eV}$, and Mie resonance energies by $5.67 \mathrm{eV}$.

\section{THEORY AND COMPARISON WITH EXPERIMENT}

As pointed out in the Introduction, we extend our previous treatment ${ }^{7}$ of the dispersion of electronic surface excitations at jellium surfaces in order to account for three important effects related to the crystal potential.

(1) Instead of the Lang-Kohn jellium model, we use the stabilized jellium model of Perdew et al. ${ }^{10}$ The virtue of this model is that it includes the ionic pseudopotentials in an average manner. Despite its simplicity, this model gives quite accurate work functions and surface energies. This improvement also influences the static and dynamic response properties, since the improved work function modifies also the unoccupied continuum and, consequently, the excitation properties of the surface. In the case of $\mathrm{Hg}$, the increase of the work function by about $0.4 \mathrm{eV}$ compared to the standard jellium model leads to a stiffer, less polarizable density profile. The resulting reduction of $\operatorname{Re} d\left(\omega_{s}\right)$ contributes directly to the flattening of the surface-plasmon dispersion curve. Additional refinements of the stabilized jellium model might, in principle, also allow the study of the crystal face dependence of the dynamical response properties.

(2) We incorporate the influence of bulk optical interband transitions involving the conduction band by evaluating the dynamical surface response at complex frequencies. The imaginary part $\gamma(\omega)$ at a given frequency $\omega$ is derived from the imaginary component $\varepsilon_{2}(\omega)$ of the bulk dielectric constant by using the relation $\gamma=0.5 \varepsilon_{2}(\omega) \omega^{3} / \omega_{p}^{2}$. In the small- $q_{\|}$limit, this treatment yields the correct surface-plasmon broadening as obtained from the expression $g(q=0, \omega)=[\varepsilon(\omega)-$ $1] /[\varepsilon(\omega)+1]$ [see Eq. (2)]. At larger $q_{\|}$, however, this incorporation of bulk absorption processes would not be realistic, since the induced surface-plasmon field decays exponentially into the metal. Thus, damping of the surface plasmon via bulk interband transitions must diminish at finite $q_{\|}$. In order to take this effective reduction of volume decay channels qualitatively into account, we use $\gamma\left(\omega, q_{\|}\right)=\gamma(\omega) \exp (-q a)$, where the length $a \approx 3 \AA$ is chosen to get an approximate representation of the $q$ dependence of the measured surface-plasmon FWHM.

It is well known ${ }^{36}$ that the treatment of damping in the relaxation-time approximation should be corrected in order to conserve the local electron density. In the case of a three-dimensional homogeneous electron gas, corrections of the order of $\gamma / \omega$ are found. An extension to semi-infinite metals has not yet been attempted. Since it would necessarily have to address the $q$-dependent balance of bulk and surface broadening mechanisms, the question arises whether such a distinction at finite $q$ is meaningful or whether a rigorous microscopic treatment must be performed that deals with bulk and surface processes on an equal footing.

(3) The frequency of the $\mathrm{Hg}$ surface plasmon lies about $0.7 \mathrm{eV}$ below the onset of interband transitions involving the $5 d$-core levels. To include the effect of these cores on the plasmon dispersion in an approximate way, we replace them by a semi-infinite polarizable medium, which partially screens the self-consistent Coulomb interaction 
among the $\mathrm{Hg} 6 s$ electrons. This $s$ - $d$ polarization had recently been considered ${ }^{11,12}$ in the context of the $\mathrm{Ag}$ surface-plasmon dispersion.

With these three modifications, the electronic surface excitations were calculated both within the TDLDA and the RPA. The induced surface charge density is given by the expression $\left(q \equiv q_{\|}\right)$

$$
\delta n(z, q, \omega)=\int d z^{\prime} \chi\left(z, z^{\prime}, q, \omega+i \gamma\right) \phi_{\mathrm{scf}}\left(z^{\prime}, q, \omega\right)
$$

where $\chi$ is the independent-particle susceptibility at the complex frequency (see above). The stabilized jellium model yields an improved ground-state surface barrier potential from which the bound states and the Green's functions are derived that are used in the construction of $\chi . \phi_{\text {scf }}$ is the self-consistently screened complex local potential. In the TDLDA, this potential consists of a superposition of external, electrostatic, and exchangecorrelation contributions. In the RPA, these exchangecorrelation terms are neglected.

In the presence of the polarizable medium representing the core levels, the electrostatic potential satisfies the modified Poisson equation:

$$
\left(\frac{d^{2}}{d z^{2}}-q^{2}\right) \phi(z, q, \omega)=-4 \pi \frac{\delta n(z, q, \omega)}{\varepsilon_{b}(\omega) \Theta\left(z_{d}-z\right)+1}
$$

where $\Theta(z \geq 0)=1$ and $\Theta(z<0)=0$. Thus, the polarizable medium is assumed to extend up to the distance $z_{d}$ from the surface. For simplicity, we will mainly put this boundary at the jellium edge $\left(z_{d}=0\right)$. Slight variations of this position do not alter the qualitative behavior of the electronic surface excitations. Additional details of concerning the treatment of the core polarization can be found in Ref. 12. As mentioned above, this model provides a qualitative explanation for the positive surfaceplasmon dispersion observed on Ag. Simply stated, the reason for this sign reversal compared to the dispersion at simple metal surfaces is that, because of the spill out of the $s$-electron density (the $d$ electrons are more localized), the mutual $s$ - $d$ polarization is weaker than in the bulk. This effect becomes more important with increasing parallel momentum, since the penetration depth of electric field associated with the surface plasmon decreases exponentially. Thus, the fluctuating charge is progressively less screened via the $d$ states, i.e., its frequency shifts upwards in the direction of the unscreened plasmon dispersion. Self-consistent TDLDA calculations ${ }^{11,12}$ show that this mechanism is indeed strong enough to give a positive dispersion in qualitative agreement with the data. ${ }^{20-22}$ Moreover, since the $d$ band is treated as a polarizable half-space, the $q=0$ limit of the predicted surface-plasma frequency coincides with the experimentally observed value. Of course, because of the neglect of the discrete lattice structure, the crystal face dependence of the dispersion and its anisotropy on $\mathrm{Ag}(110)$ cannot be addressed within this model. As shown in Fig. 1, the $d$-band contribution to the dielectric function of $\mathrm{Ag}$ near the surface plasmon is about 4 , while in the case of $\mathrm{Hg}$, it is only about 1 . Thus, in $\mathrm{Hg}$ the effect discussed above should be considerably weaker.

The three modifications (improved surface barrier, broadening due to interband transitions, and core polarization) that we have introduced in order to account for the most basic average effects of the crystal potential on the ground-state and surface response properties, were chosen with the aim of maintaining the one-dimensional simplicity of our previous response treatment of standard jellium surfaces. Since full three-dimensional response calculations for realistic metal surfaces are considerably more involved, ${ }^{37}$ this computational advantage is appreciable, in particular, since the qualitative physics of these lattice effects is reproduced reasonably well.

In Fig. 5, we show surface excitation spectra for $\mathrm{Hg}$ as calculated within the TDLDA and lattice effects included as described above. At small $q$, the surface-plasmon line first shifts to slightly lower frequencies until it reaches a minimum at about $0.1 \AA^{-1}$. At larger momenta it disperses upwards. The linewidth is finite at all parallel momenta; in the limit of small $q$ it approaches the bulk value related to $\varepsilon_{2}\left(\omega_{\mathrm{sp}}\right)$. No evidence is found in

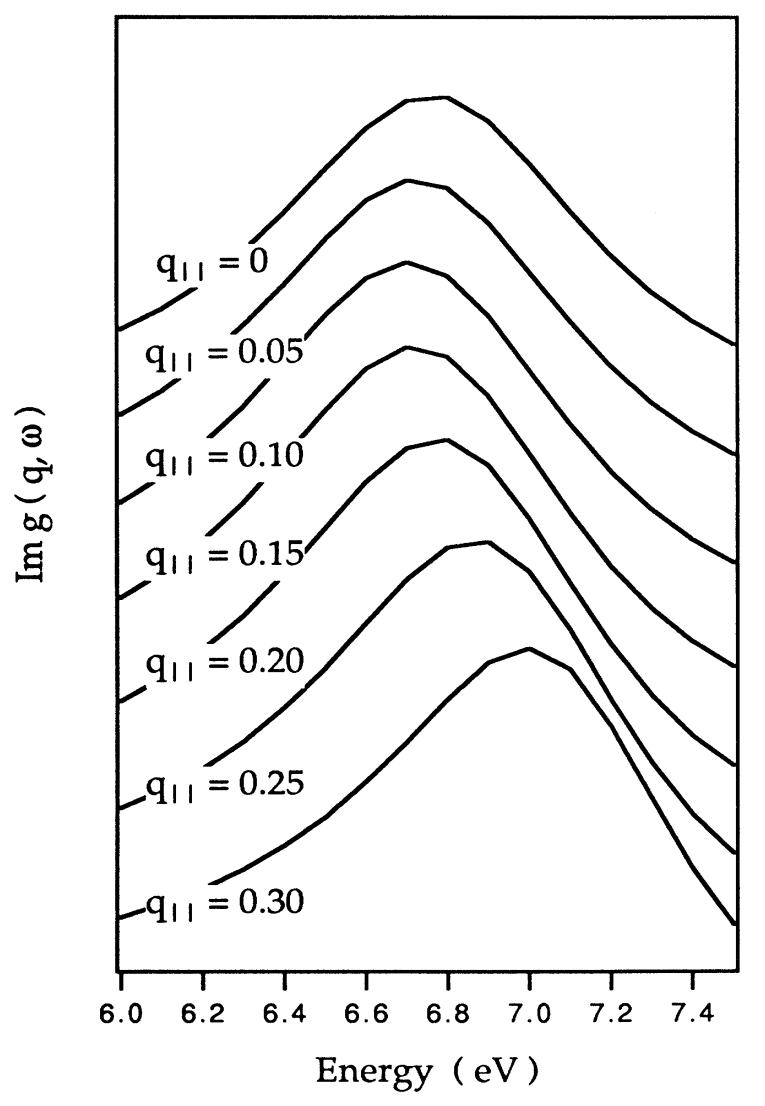

FIG. 5. Calculated surface excitation spectra of $\mathrm{Hg}$ at $q_{\|}=0.0,0.05, \ldots, 0.30 \AA^{-1}$ (from the top; the curves are vertically displaced by 1 a.u. for better readability). Crystal effects are taken into account in three aspects: stabilized jellium model, broadening due to interband transitions, and core polarization. The response is calculated within the TDLDA. 
these spectra for the multipole surface-plasmon mode. Since $\mathrm{Hg}$ has a relatively high electron density, this mode should in any case be weaker than for the alkali metals. The three lattice effects considered here should diminish the spectral weight of this mode even further: Thus, the improved work function in our model makes the density profile stiffer and less polarizable. It, therefore, becomes more difficult to excite this mode. Also, the reduced strength of the Coulomb interaction, which is partially screened by the core levels, decreases the amplitude of surface excitations. Broadening due to interband transitions involving the conduction band presumably also leads to additional weakening of the multipole mode.

In order to illustrate the lattice effects on these spectra, we show in Fig. 6 the dispersion of the energy and width of the $\mathrm{Hg}$ surface plasmon and compare them to the corresponding dispersions obtained for a standard jellium system with $r_{s}=2.65$. Also plotted are the experimental results that were already presented in Fig. 3. The shallow $\mathrm{Hg} 5 d$-core levels are seen to cause an overall

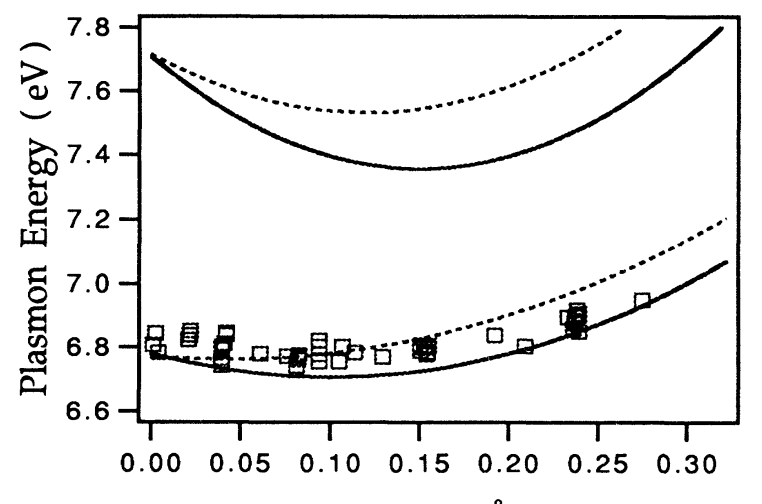

$\mathrm{q}_{11}(1 / \AA)$

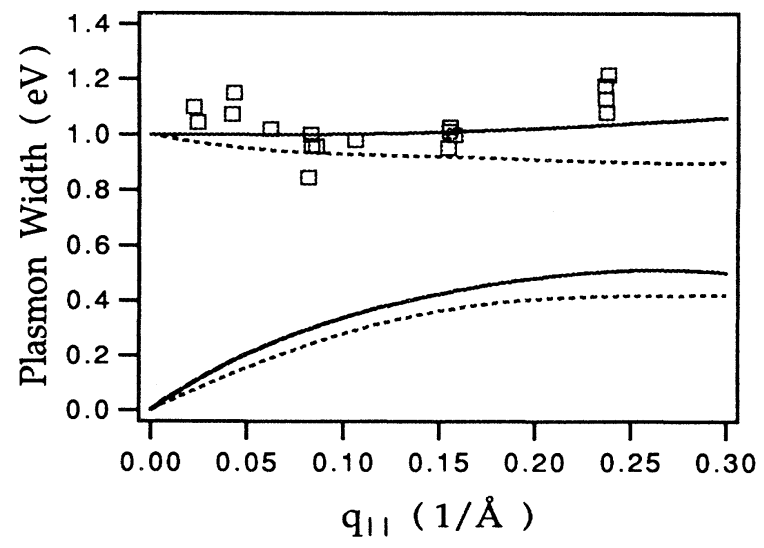

FIG. 6. Comparison of calculated and measured $\mathrm{Hg}$ surface-plasmon dispersion: (a) energy and (b) linewidth. Stabilized jellium model in the presence of polarizable core medium, plus broadening due to interband transitions. Both TDLDA (solid line) and RPA (dotted line) results are plotted. The upper curves in (a), and the lower curves in (b) are the corresponding results for the standard jellium model. lowering of the surface-plasma frequencies by about $12 \%$ relative to the free-electron values. The crystal potential leads to a significant flattening of the momentum dispersion compared to the dispersion obtained for the simple metals. The linear coefficient of the dispersion is still negative, but much smaller than in the absence of the core levels. Accordingly, the minimum of the dispersion curve is shifted to smaller momenta. As in the case of standard jellium, the RPA response gives slightly higher frequencies at finite $q$, because of the absence of exchangecorrelation terms in the induced potential. Inward shifts of the boundary of the polarizable medium representing the core levels would produce an additional slight upward distortion of both calculated dispersion curves. The overall dispersion of the $\mathrm{Hg}$ surface-plasmon frequency is seen to be in remarkable agreement with the experimental results.

The width of the surface plasmon is plotted in Fig. 6(b). The broadening obtained within the standard jellium model is seen to be far too small. At small parallel wave vectors, the experimental results are quite well reproduced by the damping derived from the imaginary part $\varepsilon_{2}(\omega)$ of the bulk dielectric function. At larger $q_{\|}$, the calculated total width remains roughly constant, because we assume the bulk contribution to decrease with $q$ as discussed above. Without this $q$-dependent reduction of bulk decay channels the total width would increase and become too large compared to the experimental observation.

It is conceivable that the slight decrease of the measured width at small $q$ [which has also been observed $\mathrm{Mg}(0001)$ (Ref. 15) and $\mathrm{Ag}(111)$ (Ref. 35)] could be related to a more rapid switching off of bulk interband transitions before broadening, due to intrinsic surface excitations, becoming the dominant mechanism at larger $q$. The total width then should pass through a minimum. More experimental data and a better theoretical understanding of the interplay between surface and bulk processes in the surface-plasmon damping would be desirable.

The main message of these results is that the surfaceplasmon dispersion depends quite sensitively on both the ground-state electronic properties and the dynamical response. In the present case of $\mathrm{Hg}$, the dispersion is still negative at small momenta, but with a considerably reduced linear slope compared to that of a jellium surface in the absence of band structure and core polarization effects. Thus, $\mathrm{Hg}$ can be considered as an intermediate case between the simple metals and Ag.

We conclude this section by briefly commenting on possible effects arising from dynamical corrections to the induced exchange-correlation potential. As shown by Gross and Kohn, ${ }^{38}$ such corrections give a weakening of the real part of the effective Coulomb interaction and a finite imaginary contribution. Explicit calculations of this effect for standard jellium surfaces ${ }^{39}$ show that the resulting dispersion lies in between the TDLDA and RPA dispersion curves. In the present case of $\mathrm{Hg}$, improvements of the adiabatic TDLDA in the direction of frequencydependent exchange-correlation potentials would, therefore, lead to a similar agreement with the data. 


\section{CONCLUSION}

The energy and width of the surface plasmon of $\mathrm{Hg}$ has been measured and compared to theoretical predictions based on a model that goes beyond previous treatments of electronic surface excitations in three crucial ways: (i) The average ionic pseudopotential of the crystal is included by utilizing the stabilized jellium model. Compared to the usual Lang-Kohn model, this improvement gives better electronic density profiles, which are of fundamental importance for the surface polarization properties, in particular, for high-density metals such as $\mathrm{Hg}$ considered here. (ii) The considerable line broadening that has been observed in all ELS measurements of surface-plasmon dispersions, is partially caused by bulk interband transitions involving the conduction band, in particular, at small momental where intrinsic surfaceinduced broadening becomes weak. We have taken the bulk-induced broadening into account by evaluating the surface response at complex frequencies, with the imaginary parts determined by $\varepsilon_{2}(\omega)$. This simple approach works well at small $q$. At larger $q$, this plasmon broadening due to bulk interband transitions is assumed to diminish exponentially because of the smaller penetration depth of the induced electric field. (iii) The presence of the shallow core levels of $\mathrm{Hg}$ is included by replacing them via a polarizable half-space. This effect leads to a $q$-dependent reduction of the mutual $s$ - $d$ polarization. Thus, the cores not only cause an overall lowering of the excitation frequencies, but an upward distortion of the momentum dispersion curve.

Remarkable agreement is presented between the $q_{\|}$dependence of the $\mathrm{Hg}$ surface-plasmon energy and width, and the $1 / R$ dependence of the Mie resonance in $\mathrm{Hg}$ clusters. Existing theoretical approaches support such a correspondence in the small- $q /$ small- $1 / R$ limit. The circumstance that this correspondence holds also beyond this range is very intriguing and requires better theoretical understanding.

We finally stress again that there is not a conflict between excitation spectra $(\operatorname{Im} g(q, \omega))$ calculated at finite $q_{\|}$and the long-wavelength behavior characterized by the function $d(\omega)$. As long as both quantities are based on the same physical assumptions, they provide a consistent picture. Thus, if lattice effects such as interband transitions, core polarization, etc. are considered, they must be incorporated at all stages. Combinations of volume dielectric functions with $d(\omega)$ parameters obtained for plain jellium models lead to uncontrolled errors in the frequency and width of the surface collective modes. These arguments apply equally to spherical particles.

\section{ACKNOWLEDGMENTS}

This work was supported by the National Science Foundation through Grants Nos. DMR-93-96059 (E.W.P.) and DMR-92-21655 (P.A.D.). Portions of this work were done at ORNL, sponsored by the Division of Materials Sciences, U.S. Department of Energy, under Contract No. DE-AC05-84OR21400 with Martin Marietta Energy Systems, Inc. One of the authors (A.L.) acknowledges extensive e-mail interchanges with Bill Schaich on the consistent treatment of lattice effects in the small- $q$ limit.
* Present address: Oak Ridge National Laboratory, Oak Ridge, TN 37831-6057. Electronic address: bkim@solid.ssd.ornl.gov

${ }^{1}$ R. H. Ritchie, Phys. Rev. 106, 874 (1957).

2 J. Harris and A. Griffin, Phys. Lett. 34A, 51 (1971).

${ }^{3}$ F. Flores and F. Garcia-Moliner, Solid State Commun. 11, 1295 (1972).

${ }^{4}$ P. J. Feibelman, Phys. Rev. Lett. 30, 975 (1973); Phys. Rev. B 9, 5077 (1974).

${ }^{5}$ N. D. Lang and W. Kohn, Phys. Rev. B 1, 4555 (1970).

${ }^{6}$ K.-D. Tsuei, E. W. Plummer, and P. J. Feibelman, Phys. Rev. Lett. 63, 2256 (1989).

${ }^{7}$ K.-D. Tsuei, E. W. Plummer, A. Liebsch, K. Kempa, and P. Bakshi, Phys. Rev. Lett. 64, 44 (1990); K.-D. Tsuei, E. W. Plummer, A. Liebsch, E. Pehlke, K. Kempa, and P. Bakshi, Surf. Sci. 247, 302 (1991).

${ }^{8}$ A. Zangwill and P. Soven, Phys. Rev. A 21, 1561 (1980).

${ }^{9}$ H. Haberland, B. von Issendorff, J. Yufeng, and T. Kolar, Phys. Rev. Lett. 69, 3212 (1992).

${ }^{10}$ J. P. Perdew, H. Q. Tran, and E. Smith, Phys. Rev. B 42, 11627 (1990); J. H. Rose and H. B. Shore, ibid. 43, 11605 (1991).

${ }^{11}$ A. Liebsch, Phys. Rev. Lett. 71, 145 (1993).

12 A. Liebsch, Phys. Rev. B 48, 11317 (1993).

${ }^{13}$ B. N. J. Persson and E. Zaremba, Phys. Rev. B 30, 5669
(1984)

${ }^{14}$ P. J. Feibelman, Prog. Surf. Sci. 12, 287 (1982).

${ }^{15}$ P. T. Sprunger, G. M. Watson, and E. W. Plummer, Surf. Sci. 269/270, 551 (1992).

${ }^{16}$ H. Ehrenreich and H. R. Philipp, Phys. Rev. 128, 1622 (1962).

${ }^{17}$ R. E. Palmer and S. E. Schnatterly, Phys. Rev. B 4, 2329 (1971).

${ }^{18}$ E. D. Palik, Handbook of Optical Constants of Solids II (Academic, San Diego, 1991), pp. 467-469.

${ }^{19}$ P. B. Johnson and R. W. Cristy, Phys. Rev. B 6, 4370 (1972).

${ }^{20}$ R. Contini and J. M. Layet, Solid State Commun. 64, 1179 (1987).

${ }^{21}$ M. Rocca, M. Lazzarino, and U. Valbusa, Phys. Rev. Lett. 69, 2122 (1992); Geunseop Lee, P. T. Sprunger, and E. W. Plummer, Surf. Sci. Lett. 286, L547 (1993).

${ }^{22} \mathrm{M}$. Rocca and U. Valbusa, Phys. Rev. Lett. 64, 2398 (1990).

${ }^{23}$ A. Liebsch and W. L. Schaich (unpublished).

${ }^{24}$ P. Apell and $\AA$. Ljungbert, Solid State Commun. 44, 1367 (1982).

${ }^{25}$ P. Apell and $\AA$. Ljungbert, Phys. Scr. 26, 113 (1982).

${ }^{26}$ G. Mie, Ann. Phys. (Leipzig) 25, 377 (1908).

${ }^{27}$ C. Bréchignac, Ph. Cahuzac, N. Kebaili, J. Leygnier, and 
A. Sarfati, Phys. Rev. Lett. 68, 3916 (1992).

${ }^{28} \mathrm{~J}$. Tiggesbäumker, L. Köller, K.-H. Meiwes-Broer, and A. Liebsch, Phys. Rev. A 48, R1749 (1993).

${ }^{29}$ C. Bréchignac, Ph. Cahuzac, N. Kebaili, J. Leygnier, and A. Sarfati, Phys. Rev. Lett. 68, 3916 (1992).

${ }^{30}$ T. Reiners, C. Ellert, M. Schmidt, and H. Haberland, Phys. Rev. Lett. 74, 1558 (1995).

${ }^{31}$ W. J. Choyke, S. H. Vosko, and T. W. O'Keeffe, Solid State Commun. 9, 361 (1971).

${ }^{32}$ W. E. Mueller and J. C. Thompson, Phys. Rev. Lett. 23, 1037 (1969).
${ }^{33}$ A. R. Krauss and R. Gomer, Phys. Rev. B 13, 3419 (1976); Y. B. Zhao and R. Gomer, Surf. Sci. 271, 85 (1992).

${ }^{34}$ A. Liebsch, Phys. Rev. B 36, 7378 (1987).

${ }^{35} \mathrm{M}$. Rocca (private communication); Geunseop Lee et al. (unpublished).

${ }^{36}$ N. D. Mermin, Phys. Rev. B 1, 2362 (1970).

${ }^{37} \mathrm{H}$. Ishida and A. Liebsch, Phys. Rev. B 45, 6171 (1992).

${ }^{38}$ E. K. U. Gross and W. Kohn, Phys. Rev. Lett. 55, 2850 (1985).

${ }^{39}$ E. Pehlke and A. Liebsch (unpublished). 